\title{
Auxiliary Principle and Iterative Algorithm for a System of Generalized Nonlinear Mixed Quasi- variational-like Inequalities
}

\author{
Yali Zhao*, Yuanyuan Sun and Qian Ji \\ College of Mathematics and Physics, Bohai University, Jinzhou, Liaoning 121013, P. R. china \\ ${ }^{*}$ Corresponding author
}

\begin{abstract}
In this paper, the auxiliary principle technique is extended to study a system of generalized nonlinear mixed quasivariational-like inequalities in Hilbert spaces. First, we establish the existence of solutions of the corresponding system of auxiliary generalized nonlinear mixed qusi-variational-like inequalities. Then based on the existence result, we construct a new iterative algorithm. Finally, both the existence of solutions of the original problem and the convergence of iterative sequences generated by the algorithm are proved. Our results improve and extend some known results.
\end{abstract}

Keywords-auxiliary principle; existence; iterative algorithm; system of generalized nonlinear mixed quasi-variational-like inequalities; convergence

\section{INTRODUCTION AND PRELIMINARIES}

Throughout the paper, let $I=\{1,2\}$ be an index set, for each $i \in I$, let $H_{i}$ be a real Hilbert spaces with inner product $\langle\cdot, \cdot\rangle_{i}$ and norm $\|\cdot\|_{i}$, and $2^{H_{i}}$ be the family of all nonempty subset of $H_{i}$. Let $K_{i}: H_{i} \rightarrow 2^{H_{i}}$ be a set-valued mapping such that for each $x_{i} \in H_{i}, K_{i}(x)$ is a nonempty closed convex subset of $H_{i}$ Let $N_{i}, \eta_{i}: H_{i} \times H_{i} \rightarrow H_{i}$,

$M_{i}: H_{1} \times H_{2} \rightarrow H_{i}, A_{i}, B_{i}: H_{i} \rightarrow H_{i}, C_{i}: H_{1} \rightarrow H_{1}, D_{i}: H_{2} \rightarrow H_{2}$ be nonlinear single-valued mappings. We consider the following system of generalized nonlinear mixed quasivariational-like inequalities (for short, denoted by SGNMQVLI): Find $(u, v) \in K_{1}(u) \times K_{2}(v)$ such that

$\left\{\begin{array}{l}\left\langle N_{1}\left(A_{1} x_{1}, B_{1} x_{1}\right)+M_{1}\left(C_{1} x_{1}, D_{1} y\right), \eta_{1}(u, x)\right\rangle_{1}+b_{1}(x, u)-b_{1}(x, u) \geq 0, \forall u \in K_{1}(x), \\ \left\langle N_{2}\left(A_{2} y, B_{2} y\right)+M_{2}\left(C_{2} x, D_{2} y\right), \eta_{2}(v, y)\right\rangle_{2}+b_{2}(y, v)-b_{2}(y, y) \geq 0, \forall v \in K_{2}(y) .\end{array}\right.$

where $b_{i}: H_{i} \times H_{i} \rightarrow R$ is a bifunction, which has the following properties:

(i) $b_{i}(\cdot, \cdot)$ is linear in the first argument;

(ii) $b_{i}(\cdot, \cdot)$ is convex in the second argument; (iii) $b_{i}(\cdot, \cdot)$ is bounded, that is, there exists a constant $C_{i}>0$ such that $b_{i}\left(u_{i}, v_{i}\right) \leq C_{i}\left\|u_{i}\right\|\left\|v_{i}\right\|_{i}, \forall u_{i}, v_{i} \in H_{i}$;

(iv) $b_{i}\left(u_{i}, v_{i}\right)-b_{i}\left(u_{i}, w_{i}\right) \leq b_{i}\left(u_{i}, v_{i}-w_{i}\right), \forall u_{i}, v_{i}, w_{i} \in H_{i}$. In many important applications, $K_{1}(u)$ and $K_{2}(v)$ have the

following forms [1, 3]:

$$
K_{1}(u)=m_{1}(u)+K_{1}, \forall u \in H_{1}, K_{2}(v)=m_{2}(v)+K_{2}, \forall v \in H_{2},
$$

where $m_{i}: H_{i} \rightarrow H_{i}$ is a single-valued mapping and $K_{i}$ is a nonempty closed convex subset of $H_{i}$.

Noting that if $H_{1}=R^{n}, H_{2}=R^{m}, K_{1}(x)=K_{1}, K_{2}(y)=K_{2}$, $N_{1}=N_{2}=b_{1}=b_{2}=0, C_{1}=C_{2}=I_{H_{1}}, D_{1}=D_{2}=I_{H_{2}}, \eta_{1}=u-x$, $\eta_{2}=v-y, \forall x, u \in H_{1}, y, v \in H_{2}$, where $I_{H_{1}}$ and $I_{H_{2}}$ are identity mappings on $H_{1}$ and $H_{2}$, respectively, then SGNMQVLI(1) reduces to the following system of variational inequalities (for short, denoted by SVI): Find $(u, v) \in K_{1} \times K_{2}$ such that

$$
\left\{\begin{array}{l}
\left\langle M_{1}(u, v), w-u\right\rangle_{1} \geq 0, \forall w \in K_{1}, \\
\left\langle M_{2}(u, v), z-v\right\rangle_{2} \geq 0, \forall z \in K_{2} .
\end{array}\right.
$$

SVI(3) was introduced and studied by Zhao et al. [3], in which they employed the Brouwer fixed point theorem to obtain some existence results for SVI(3), moreover, by projection technique, they established the existence and uniqueness theorem for SVI(3) and suggested an iterative algorithm and analysised convergence of the algorithm. But in SGNMQVLI(1), the two convex sets depend on the solutions implicitly or explicitly, and $b_{i}$ is a nonlinear mapping, so the projection technique cannot be applied to it. This fact motivated many authors to develop the auxiliary principle technique to study the existence of solutions of generalized mixed type quasi-variational inequalities and also to develop a large number of numerical methods for solving various variational inequalities, 
comlementarity problems and optimization problems. The auxiliary principle technique was first introduced by Glowinski et al. [4]. Recently, auxiliary principle technique has especially attracted the attention of scholars in the area of variational inequality theory, for details, see [5-8] and the references therein.

Motivated and inspired by the above research work, in this paper, we introduce and study SGNMQVLI(1) in Hilbert spaces. By applying the auxiliary principle technique, we show the existence and uniqueness theorem of solution for the corresponding auxiliary principle relative to SGNMQVLI (1) by minimizing sequence method. For finding the approximate solutions of SGNMQVLI (1), we suggest an iterative algorithm by the auxiliary problem. Under certain conditions, we obtain the existence result of solution for SGNMQVLI (1) and prove the convergence of iterative sequences generated by the iterative algorithm. Our results improve and generalized many known results.

In order to obtain our main results, we first recall some concept and assumption.

Definition 1.1 Let $H$ be a real Hilbert space, $A, B: H \rightarrow H$ $N, \eta: H \times H \rightarrow H$, be single-valued mappings.

(1) $\eta$ is said to be $\delta$-Lipschitz continuous, if there exists a constant $\delta>0$ such that

$$
\|\eta(x, y)\| \leq \delta\|x-y\|, \forall x, y \in H
$$

(2) $A$ is said to be $\omega$ - Lipschitz continuous, if there exists a constant $\omega>0$ such that

$$
\|A x-A y\| \leq \omega\|x-y\|, \forall x, y \in H ;
$$

(3) $N$ is said to be $(\mu, v)$ - Lipschitz continuous, if there exists a pair of constants $\mu, v>0$ such that

$$
\begin{gathered}
\left\|N\left(x_{1}, y_{1}\right)-N\left(x_{2}, y_{2}\right)\right\| \leq \mu\left\|x_{1}-x_{2}\right\|+v\left\|y_{1}-y_{2}\right\|, \\
\forall x_{1}, x_{2}, y_{1}, y_{2} \in H ;
\end{gathered}
$$

(4) $N$ is said to be $\xi$ - relaxed Lipschitz with respect to $A$ and $B$ in the first argument and second argument, if there exists a constants $\xi>0$ such that

$$
\langle N(A x, B x)-N(A y, B y), x-y\rangle \leq-\xi\|x-y\|^{2}, \forall x, y \in H
$$

(5) $N$ is said to be $\alpha$-Lipschitz continuous in the first argument, if there exists a constant $\alpha>0$ such that

$$
\|N(x, z)-N(y, z)\| \leq \alpha\|x-y\|, \forall x, y, z \in H ;
$$

In a similar way, we can define the Lipschitz continuity of $N$ in the second argument.
Assumption1.1 The mapping $\eta_{i}, N_{i}: H_{i} \times H_{i} \rightarrow H_{i}$, $A_{i}, B_{i}: H_{i} \rightarrow H_{i}, C_{i}: H_{1} \rightarrow H_{1}, D_{i}: H_{2} \rightarrow H_{2}$ satisfies the following conditions:

(1) $\eta_{i}\left(u_{i}, v_{i}\right)=\eta_{i}\left(u_{i}, z_{i}\right)+\eta_{i}\left(z_{i}, v_{i}\right), \forall u_{i}, v_{i}, z_{i} \in H_{i}$;

(2) $\eta_{i}\left(u_{i}+v_{i}, w_{i}\right)=-\eta_{i}\left(w_{i}-u_{i}, v_{i}\right), \forall u_{i}, v_{i}, w_{i} \in H_{i}$;

(3) The functions

$$
\begin{aligned}
& u \rightarrow\left\langle N_{1}\left(A_{1} x, B_{1} x\right)+M_{1}\left(C_{1} x, D_{1} y\right), \eta_{1}(u, x)\right\rangle_{1}, \\
& v \rightarrow\left\langle N_{2}\left(A_{2} y, B_{2} y\right)+M_{2}\left(C_{2} x, D_{2} y\right), \eta_{2}(v, y)\right\rangle_{2}
\end{aligned}
$$

are both continuous and linear for all $(u, v) \in H_{1} \times H_{2}$.

Remark1.1 It follows from Assumption1.1 (1) that

$$
\eta_{i}\left(u_{i}, u_{i}\right)=0, \eta_{i}\left(u_{i}, v_{i}\right)=-\eta_{i}\left(v_{i}, u_{i}\right), \phi о \rho \alpha \lambda \lambda u_{i}, v_{i} \in H_{i}
$$

\section{AuXiliary Problem And Iterative Algorithm}

For each $i \in I$, given $(u, v) \in K_{1}(u) \times K_{2}(v)$, we consider the following problem:

find $(p, q) \in K_{1}(u) \times K_{2}(v)$ such that

$$
\begin{aligned}
\langle p, u-p\rangle_{1} \geq & \langle x, u-p\rangle_{1}-\rho_{1}\left\langle N_{1}\left(A_{1} x, B_{1} x\right)+M_{1}\left(C_{1} x, D_{1} y\right), \eta_{1}(u, p)\right\rangle_{1} \\
& +\rho_{1}\left[b_{1}(x, p)-b_{2}(x, u)\right], \forall u \in K_{1}(x)
\end{aligned}
$$

$$
\begin{aligned}
\langle q, v-q\rangle_{2} \geq & \langle y, v-q\rangle_{2}-\rho_{2}\left\langle N_{2}\left(A_{2} y, B_{2} y\right)+M_{1}\left(C_{2} x, D_{2} y\right), \eta_{2}(v, q)\right\rangle_{2} \\
& +\rho_{2}\left[b_{2}(y, q)-b_{2}(y, v)\right], \forall v \in K_{2}(y),
\end{aligned}
$$

where $\rho_{1}, \rho_{2}>0$ are constants. This problem is called the system of auxiliary generalized nonlinear mixed quasivariational-like inequalities, for short, denoted by SAGNMQVLI (4) related to SGNQVLI (1).

Theorem 2.1 For each $i \in I$, let $K_{i}: H_{i} \rightarrow 2^{H_{i}}$ be set-valued mapping such that for each $x_{i} \in H_{i}, K_{i}\left(x_{i}\right)$ is a nonempty closed convex subset of $H_{i} . \quad N_{i}, \eta_{i}: H_{i} \times H_{i} \rightarrow H_{i}, M_{i}: H_{1} \times H_{2} \rightarrow H_{i}$, $A_{i}, B_{i}: H_{i} \rightarrow H_{i}, C_{i}: H_{1} \rightarrow H_{1}, D_{i}: H_{2} \rightarrow H_{2}$ be nonlinear singlevalued mappings, and $b_{i}: H_{i} \times H_{i} \rightarrow R$ be a bifunction such that for each given $(x, y) \in H_{1} \times H_{2}$, the functions $u \mapsto b_{1}(x, u)$ and

$v \mapsto b_{2}(y, v)$ are proper convex and lower semicontinuous. If Assumption1.1 holds, then for any given $(x, y) \in H_{1} \times H_{2}$, define the functions $J_{1}: K_{1}(x) \rightarrow R$ and $J_{2}: K_{2}(y) \rightarrow R$ as follows:

$$
J_{1}(u)=\frac{1}{2}\langle u, u\rangle_{1}+j_{1}(u), \quad J_{2}(v)=\frac{1}{2}\langle v, v\rangle_{2}+j_{2}(v),
$$

Where 
$j_{1}(u)=\rho_{1}\left\langle N_{1}\left(A_{1} x, B_{1} x\right)+M_{1}\left(C_{1} x, D_{1} y\right), \eta_{1}(u, x)\right\rangle_{1}+\rho_{1} b_{1}(x, u)-\langle x, u\rangle_{1}$, $j_{2}(v)=\rho_{2}\left\langle N_{2}\left(A_{2} y, B_{2} y\right)+M_{2}\left(C_{2} x, D_{2} y\right), \eta_{2}(v, y)\right\rangle_{2}+\rho_{2} b_{2}(y, v)-\langle y, v\rangle_{2}$.

Then we have:

(i) $J_{1}$ has a unique minimum point $p \in K_{1}(x)$, and $J_{2}$ has a unique minimum point $q \in K_{2}(y)$.

(ii) $J_{1}$ and $J_{2}$ have unique minimum points $p \in K_{1}(x)$ and $q \in K_{2}(y)$, respectively, if and only if $(p, q)$ is a unique solution of SAGNMQVLI (4).

Proof Similarly argument in Theorem 2.1[8], the conclusions are immediately obtained, so are omitted, completing the proof.

Based on Theorem 2.1, we suggest an iterative algorithm for solving SGNQVLI (1).

Algorithm 2.1 For given $\left(u_{0}, v_{0}\right) \in H_{1} \times H_{2}$, let the sequence $\left\{\left(u_{n}, v_{n}\right)\right\} \in K_{1}\left(u_{n}\right) \times K_{2}\left(v_{n}\right)$ satisfies the following conditions:

$$
\begin{aligned}
\left\langle x_{n+1}, u\right. & \left.-x_{n+1}\right\rangle_{1} \geq\left\langle x_{n}, u-x_{n+1}\right\rangle_{1} \\
& -\rho_{1}\left\langle N_{1}\left(A_{1} x_{n}, B_{1} x_{n}\right)+M_{1}\left(C_{1} x_{n}, D_{1} y_{n}\right), \eta_{1}\left(u, x_{n+1}\right)\right\rangle_{1} \\
& +\rho_{1}\left[b_{1}\left(x_{n}, x_{n+1}\right)-b_{1}\left(x_{n}, u\right)\right], \forall u \in K_{1}\left(x_{n+1}\right) ; \\
\left\langle y_{n+1}, v-\right. & \left.y_{n+1}\right\rangle_{2} \geq\left\langle y_{n}, v-y_{n+1}\right\rangle_{2} \\
& -\rho_{2}\left\langle N_{2}\left(A_{2} y_{n}, B_{2} y_{n}\right)+M_{2}\left(C_{1} x_{n}, D_{2} y_{n}\right), \eta_{2}\left(v, y_{n+1}\right)\right\rangle_{2} \\
& +\rho_{2}\left[b_{2}\left(y_{n}, y_{n+1}\right)-b_{2}\left(y_{n}, v\right)\right], \forall v \in K_{2}\left(y_{n+1}\right),
\end{aligned}
$$

for every $n=0,1,2,3 \cdots$, where $\rho_{1}, \rho_{2}>0$ are constants.

\section{EXISTENCE AND CONVERGENCE THEOREM}

Theorem 3.1 For each $i \in I$, let $H_{i}$ be a Hilbert space, and $K_{i}: H_{i} \rightarrow 2^{H_{i}}$ be set-valued mapping such that for each $x_{i} \in H_{i}, K_{i}\left(x_{i}\right)$ is a nonempty closed convex subset of $H_{i}$. Let $N_{i}, \eta_{i}: H_{i} \times H_{i} \rightarrow H_{i}, M_{i}: H_{1} \times H_{2} \rightarrow H_{i}, A_{i}, B_{i}: H_{i} \rightarrow H_{i}$, $C_{i}: H_{1} \rightarrow H_{1}, D_{i}: H_{2} \rightarrow H_{2}$ be nonlinear single-valued mappings. Let $m_{i}: H_{i} \rightarrow H_{i}$ satisfy (2), and $b_{i}: H_{i} \times H_{i} \rightarrow R$ be a realvalued functional satisfying the properties in Theorem 2.1 and properties (i)-(iv). Assume that following conditions are satisfied:

(1) $m_{i}$ is $\tau_{i}$-Lipschitz continuous;

(2) $N_{i}$ is $\xi_{i}$-relaxed Lipschitz with respect to $A_{i}$ and $B_{i}$ in the first and second arguments, and $N_{i}$ is $\alpha_{i}$-Lipschitz continuous in the first argument and is $\beta_{i}$-Lipschitz continuous in the second argument, respectively;
(3) $M_{i}$ is $\left(\mu_{i}, v_{i}\right)$-Lipschitz continuous and $M_{i}$ is $k_{i}$ Lipschitz continuous in the first argument and is $l_{i}$-Lipschitz continuous in the second argument, respectively;

(4) $A_{i}$ is $\omega_{i}$-Lipschitz continuous;

(5) $B_{i}$ is $\gamma_{i}$-Lipschitz continuous;

(6) $C_{i}$ is $\lambda_{i}$-Lipschitz continuous;

(7) $D_{i}$ is $\sigma_{i}$-Lipschitz continuous;

(8) $\eta_{i}$ is $\delta_{i}$-Lipschitz continuous.

If Assumption1.1 holds and there exist constants $\rho_{1}, \rho_{2}>0$ such that

$\left\{\begin{array}{l}\frac{1}{1-2 \tau_{1}}\left[1+\rho_{1} C_{1}+\delta_{1} C_{1}+\sqrt{1-2 \rho_{1} \xi_{1}+\rho_{1}^{2}\left(\alpha_{1} \omega_{1}+\beta_{1} \gamma_{1}\right)^{2}}+\rho_{1} \mu_{1} \lambda_{1}\right]+\frac{\rho_{2} \delta_{2} v_{2} \sigma_{2}}{1-2 \tau_{2}}<1 \\ \frac{1}{1-2 \tau_{2}}\left[1+\rho_{2} C_{2}+\delta_{2} C_{1}+\sqrt{1-2 \rho_{2} \xi_{2}+\rho_{2}^{2}\left(\alpha_{2} \omega_{2}+\beta_{2} \gamma_{2}\right)^{2}}+\rho_{2} \mu_{2} \lambda_{2}\right]+\frac{\rho_{1} \delta_{1} v_{1} \sigma_{1}}{1-2 \tau_{1}}<1\end{array}\right.$

Then there exists $(u, v) \in K_{1}(u) \times K_{2}(v)$ is a solution of SGNQVLI (1), and the sequence $\left\{\left(u_{n}, v_{n}\right)\right\}$ generated by Algorithm2.1 strongly converges to $(u, v)$.

Proof First, it follows from (5) in Algorithm 2.1 that, for any $w \in K_{1}\left(u_{n}\right)$,

$$
\begin{aligned}
\left\langle x_{n}, u-x_{n}\right\rangle_{1} \geq & \left\langle x_{n-1}, u-x_{n}\right\rangle_{1}-\rho_{1}\left\langle N_{1}\left(A_{1} x_{n-1}, B_{1} x_{n-1}\right)+M_{1}\left(C_{1} x_{n-1}, D_{1} y_{n-1}\right), \eta_{1}\left(u, x_{n}\right)\right\rangle_{1} \\
& +\rho_{1}\left[b_{1}\left(x_{n-1}, x_{n}\right)-b_{1}\left(x_{n-1}, u\right)\right] .
\end{aligned}
$$

and for any $z \in K_{1}\left(u_{n+1}\right)$,

$\left\langle x_{n+1}, u-x_{n+1}\right\rangle_{1} \geq\left\langle x_{n}, u-x_{n+1}\right\rangle_{1}-\rho_{1}\left\langle N_{1}\left(A_{1} x_{n}, B_{1} x_{n}\right)+M_{1}\left(C_{1} x_{n}, D_{1} y_{n}\right), \eta_{1}\left(u, x_{n+1}\right)\right\rangle_{1}$ $+\rho_{1}\left[b_{1}\left(x_{n}, x_{n+1}\right)-b_{1}\left(x_{n}, u\right)\right]$.

Adding $\left\langle-m_{1}\left(u_{n}\right), w-u_{n}\right\rangle_{1}$ to the two sides of inequality (8) and then taking $w=m_{1}\left(u_{n}\right)+u_{n+1}-m_{1}\left(u_{n+1}\right) \in K_{1}\left(u_{n}\right)$, we get

$$
\begin{aligned}
& \left\langle x_{n}-m_{1}\left(x_{n}\right), m_{1}\left(x_{n}\right)+x_{n+1}-m_{1}\left(x_{n+1}\right)-x_{n}\right\rangle_{1} \\
\geq & \left\langle x_{n-1}-m_{1}\left(x_{n}\right), m_{1}\left(x_{n}\right)+x_{n+1}-m_{1}\left(x_{n+1}\right)-x_{n}\right\rangle_{1} \\
& -\rho_{1}\left\langle N_{1}\left(A_{1} x_{n-1}, B_{1} x_{n-1}\right)+M_{1}\left(C_{1} x_{n-1}, D_{1} y_{n-1}\right), \eta_{1}\left(m_{1}\left(x_{n}\right)+x_{n+1}-m_{1}\left(x_{n+1}\right), x_{n}\right)\right\rangle_{1} \\
& +\rho_{1}\left[b_{1}\left(x_{n-1}, x_{n}\right)-b_{1}\left(x_{n-1}, m_{1}\left(x_{n}\right)+x_{n+1}-m_{1}\left(x_{n+1}\right)\right)\right] .
\end{aligned}
$$

Adding $\left\langle-m_{1}\left(u_{n+1}\right), w-u_{n+1}\right\rangle_{1}$ to the two sides of inequality (9) and then taking $w=m_{1}\left(u_{n+1}\right)+u_{n}-m_{1}\left(u_{n}\right) \in K_{1}\left(u_{n+1}\right)$, we get 


$$
\begin{aligned}
& \left\langle x_{n+1}-m_{1}\left(x_{n+1}\right), m_{1}\left(x_{n+1}\right)+x_{n}-m_{1}\left(x_{n}\right)-x_{n+1}\right\rangle_{1} \\
\geq & \left\langle x_{n}-m_{1}\left(x_{n+1}\right), m_{1}\left(x_{n+1}\right)+x_{n}-m_{1}\left(x_{n}\right)-x_{n+1}\right\rangle_{1} \\
& -\rho_{1}\left\langle N_{1}\left(A_{1} x_{n}, B_{1} x_{n}\right)+M_{1}\left(C_{1} x_{n}, D_{1} y_{n}\right), \eta_{1}\left(m_{1}\left(x_{n+1}\right)+x_{n}-m_{1}\left(x_{n}\right), x_{n+1}\right)\right\rangle_{1} \\
& +\rho_{1}\left[b_{1}\left(x_{n}, x_{n+1}\right)-b_{1}\left(x_{n}, m_{1}\left(x_{n+1}\right)+x_{n}-m_{1}\left(x_{n}\right)\right)\right] .
\end{aligned}
$$

Adding (10) and (11), by properties (i) and (iii) of $b_{i}$ and Assumption1.1 (2), we have

$$
\begin{aligned}
& \left\langle u_{n}-u_{n+1}-m_{1}\left(u_{n}\right)+m_{1}\left(u_{n+1}\right), u_{n}-u_{n+1}-m_{1}\left(u_{n}\right)+m_{1}\left(u_{n+1}\right)\right\rangle_{1} \\
\leq & \left\langle u_{n-1}-u_{n}-m_{1}\left(u_{n}\right)+m_{1}\left(u_{n+1}\right), u_{n}-u_{n+1}-m_{1}\left(u_{n}\right)+m_{1}\left(u_{n+1}\right)\right\rangle_{1} \\
+ & \rho_{1}\left\langle N_{1}\left(A_{1} u_{n-1}, B_{1} u_{n-1}\right)-N_{1}\left(A_{1} u_{n}, B_{1} u_{n}\right),\right. \\
& \left.\eta_{1}\left(m_{1}\left(u_{n}\right)+u_{n+1}-m_{1}\left(u_{n+1}\right), u_{n}\right)\right\rangle_{1} \\
+ & \rho_{1}\left\langle M_{1}\left(C_{1} u_{n-1}, D_{1} v_{n-1}\right)-M_{1}\left(C_{1} u_{n}, D_{1} v_{n}\right),\right. \\
& \left.\eta_{1}\left(m_{1}\left(u_{n}\right)+u_{n+1}-m_{1}\left(u_{n+1}\right), u_{n}\right)\right\rangle_{1} \\
- & \rho_{1}\left[b_{1}\left(u_{n-1}, u_{n}\right)-b_{1}\left(u_{n-1}, m_{1}\left(u_{n}\right)+u_{n+1}-m_{1}\left(u_{n+1}\right)\right)\right] \\
- & \rho_{1}\left[b_{1}\left(u_{n}, u_{n+1}\right)-b_{1}\left(u_{n}, m_{1}\left(u_{n+1}\right)+u_{n}-m_{1}\left(u_{n}\right)\right)\right] \\
\leq & \left\langle u_{n-1}-u_{n}-m_{1}\left(u_{n}\right)+m_{1}\left(u_{n+1}\right), u_{n}-u_{n+1}-m_{1}\left(u_{n}\right)+m_{1}\left(u_{n+1}\right)\right\rangle_{1} \\
+ & \rho_{1}\left\langle N_{1}\left(A_{1} u_{n-1}, B_{1} u_{n-1}\right)-N_{1}\left(A_{1} u_{n}, B_{1} u_{n}\right),\right. \\
& \left.\eta_{1}\left(m_{1}\left(u_{n}\right)+u_{n+1}-m_{1}\left(u_{n+1}\right), u_{n}\right)\right\rangle_{1} \\
+ & \rho_{1}\left\langle M_{1}\left(C_{1} u_{n-1}, D_{1} v_{n-1}\right)-M_{1}\left(C_{1} u_{n}, D_{1} v_{n}\right),\right. \\
& \left.\eta_{1}\left(m_{1}\left(u_{n}\right)+u_{n+1}-m_{1}\left(u_{n+1}\right), u_{n}\right)\right\rangle_{1} \\
+ & \rho_{1} b_{1}\left(u_{n}-u_{n-1}, u_{n}-m_{1}\left(u_{n}\right)-u_{n+1}+m_{1}\left(u_{n+1}\right)\right) .
\end{aligned}
$$

By property (iii) of $b_{i}$, (12) implies that

$\left\|x_{n}-x_{n+1}-m_{1}\left(x_{n}\right)+m_{1}\left(x_{n+1}\right)\right\|_{1}^{2}$

$\leq\left\|x_{n-1}-x_{n}-m_{1}\left(x_{n}\right)+m_{1}\left(x_{n+1}\right)\right\|_{1} \cdot\left\|x_{n}-x_{n+1}-m_{1}\left(x_{n}\right)+m_{1}\left(x_{n+1}\right)\right\|_{1}$

$+\left[\left\|x_{n-1}-x_{n}+\rho_{1}\left(N_{1}\left(A_{1} x_{n-1}, B_{1} x_{n-1}\right)-N_{1}\left(A_{1} x_{n}, B_{1} x_{n}\right)\right)\right\|_{1}+\right.$

$\left.\left\|x_{n-1}-x_{n}-\rho_{1}\left(M_{1}\left(C_{1} x_{n-1}, D_{1} y_{n-1}\right)-M_{1}\left(C_{1} x_{n}, D_{1} y_{n}\right)\right)\right\|_{1}\right]$

$\left\|\eta_{1}\left(m_{1}\left(x_{n}\right)+x_{n+1}-m_{1}\left(x_{n+1}\right), x_{n}\right)\right\|_{1}$

$+\rho_{1} l_{1}\left\|x_{n}-x_{n+1}\right\|_{1}\left\|x_{n}-m_{1}\left(x_{n}\right)-x_{n+1}+m_{1}\left(x_{n+1}\right)\right\|_{1}$.

It follows from Condition (6) and (13), we have

$$
\begin{aligned}
& \left\|u_{n}-u_{n+1}-m_{1}\left(u_{n}\right)+m_{1}\left(u_{n+1}\right)\right\|_{1}^{2} \\
& \leq\left\|u_{n-1}-u_{n}-m_{1}\left(u_{n}\right)+m_{1}\left(u_{n+1}\right)\right\|_{1} \cdot\left\|u_{n}-u_{n+1}-m_{1}\left(u_{n}\right)+m_{1}\left(u_{n+1}\right)\right\|_{1} \\
& +\left[\left\|u_{n-1}-u_{n}+\rho_{1}\left(N_{1}\left(A_{1} u_{n-1}, B_{1} u_{n-1}\right)-N_{1}\left(A_{1} u_{n}, B_{1} u_{n}\right)\right)\right\|_{1}+\right. \\
& \left.\quad\left\|u_{n-1}-u_{n}-\rho_{1}\left(M_{1}\left(C_{1} u_{n-1}, D_{1} v_{n-1}\right)-M_{1}\left(C_{1} u_{n}, D_{1} v_{n}\right)\right)\right\|_{1}\right] . \\
& \quad\left\|\eta_{1}\left(m_{1}\left(u_{n}\right)+u_{n+1}-m_{1}\left(u_{n+1}\right), u_{n}\right)\right\|_{1} \\
& +\rho_{1} l_{1}\left\|u_{n}-u_{n+1}\right\|\left\|u_{n}-m_{1}\left(u_{n}\right)-u_{n+1}+m_{1}\left(u_{n+1}\right)\right\|_{1} .
\end{aligned}
$$

$$
\begin{aligned}
& \left\|u_{n}-u_{n+1}\right\|_{1} \\
\leq & \left\|u_{n-1}-u_{n}\right\|_{1}+2\left\|m_{1}\left(u_{n}\right)-m_{1}\left(u_{n+1}\right)\right\|_{1} \\
& +\delta_{1}\left[\left\|u_{n-1}-u_{n}+\rho_{1}\left(N_{1}\left(A_{1} u_{n-1}, B_{1} u_{n-1}\right)-N_{1}\left(A_{1} u_{n}, B_{1} u_{n}\right)\right)\right\|_{1}\right. \\
& \left.\left.+\left\|u_{n-1}-u_{n}\right\|_{1}+\rho_{1} \| M_{1}\left(C_{1} u_{n-1}, D_{1} v_{n-1}\right)-M_{1}\left(C_{1} u_{n}, D_{1} v_{n}\right)\right) \|_{1}\right] \\
& +\rho_{1} C_{1}\left\|u_{n-1}-u_{n}\right\|_{1} .
\end{aligned}
$$

By Conditions (2), (4) and (5), we have

$$
\begin{aligned}
& \left\|x_{n-1}-x_{n}+\rho_{1}\left(N_{1}\left(A_{1} x_{n-1}, B_{1} x_{n-1}\right)-N_{1}\left(A_{1} x_{n}, B_{1} x_{n}\right)\right)\right\|_{1}^{2} \\
\leq & {\left[1-2 \rho_{1} \xi_{1}+\rho_{1}^{2}\left(\alpha_{1} \omega_{1}+\beta_{1} \gamma_{1}\right)^{2}\right]\left\|x_{n-1}-x_{n}\right\|_{1}^{2} . }
\end{aligned}
$$

By Conditions (1), (3), (6) and (7), it follows from (14) and (15) that

$$
\begin{gathered}
\left\|x_{n}-x_{n+1}\right\|_{1} \leq \frac{1}{1-2 \tau_{1}}\left\{\left[1+\rho_{1} C_{1}+\delta_{1}\left(1+\sqrt{1-2 \rho_{1} \xi_{1}+\rho_{1}^{2}\left(\alpha_{1} \omega_{1}+\beta_{1} \gamma_{1}\right)^{2}}+\rho_{1} \mu_{1} \lambda_{1}\right)\right] .\right. \\
\left.\left\|x_{n-1}-x_{n}\right\|_{1}+\rho_{1} \delta_{1} v_{1} \sigma_{1}\left\|y_{n-1}-y_{n}\right\|_{2}\right\} .
\end{gathered}
$$

And it follows from (6), for any $v \in K_{2}\left(y_{n}\right)$, we have

$$
\begin{aligned}
\left\langle y_{n}, v\right. & \left.-y_{n}\right\rangle_{2} \geq\left\langle y_{n-1}, v-y_{n}\right\rangle_{2} \\
& -\rho_{2}\left\langle N_{2}\left(A_{2} y_{n-1}, B_{2} y_{n-1}\right)+M_{2}\left(C_{2} x_{n-1}, D_{2} y_{n-1}\right), \eta_{2}\left(v, y_{n}\right)\right\rangle_{2} \\
& +\rho_{2}\left[b_{2}\left(y_{n-1}, y_{n}\right)-b_{2}\left(y_{n-1}, v\right)\right] .
\end{aligned}
$$

And, for any $z \in K_{2}\left(v_{n+1}\right)$,

$$
\begin{aligned}
\left\langle y_{n+1}, v\right. & \left.-y_{n+1}\right\rangle_{2} \geq\left\langle y_{n}, v-y_{n+1}\right\rangle_{2} \\
& -\rho_{2}\left\langle N_{2}\left(A_{2} y_{n}, B_{2} y_{n}\right)+M_{2}\left(C_{2} x_{n}, D_{2} y_{n}\right), \eta_{2}\left(v, y_{n+1}\right)\right\rangle_{2} \\
& +\rho_{2}\left[b_{2}\left(y_{n}, y_{n+1}\right)-b_{2}\left(y_{n}, v\right)\right] .
\end{aligned}
$$

Adding $\left\langle-m_{2}\left(v_{n}\right), h_{2}-v_{n}\right\rangle_{2}$ to the two sides of inequality (17) and then taking $z=m_{2}\left(v_{n}\right)+v_{n+1}-m_{2}\left(v_{n+1}\right) \in K_{2}\left(v_{n}\right)$, we get 


$$
\begin{aligned}
& \left\langle y_{n}-m_{2}\left(y_{n}\right), m_{2}\left(y_{n}\right)+y_{n+1}-m_{2}\left(y_{n+1}\right)-y_{n}\right\rangle_{2} \\
\geq & \left\langle y_{n-1}-m_{2}\left(y_{n}\right), m_{2}\left(y_{n}\right)+y_{n+1}-m_{2}\left(y_{n+1}\right)-y_{n}\right\rangle_{2} \\
& -\rho_{2}\left\langle N_{2}\left(A_{2} y_{n-1}, B_{2} y_{n-1}\right)+M_{2}\left(C_{2} x_{n-1}, D_{2} y_{n-1}\right), \eta_{2}\left(m_{2}\left(y_{n}\right)+y_{n+1}-m_{2}\left(y_{n+1}\right), y_{n}\right)\right\rangle_{2} \\
& +\rho_{2}\left[b_{2}\left(y_{n-1}, y_{n}\right)-b_{2}\left(y_{n-1}, m_{2}\left(y_{n}\right)+y_{n+1}-m_{2}\left(y_{n+1}\right)\right)\right] .
\end{aligned}
$$

Adding $\left\langle-m_{2}\left(v_{n+1}\right), z-v_{n+1}\right\rangle_{2}$ to the two sides of inequality (18) and then taking $z=m_{2}\left(v_{n+1}\right)+v_{n}-m_{2}\left(v_{n}\right) \in K_{2}\left(v_{n+1}\right)$, we get

$$
\begin{aligned}
& \left\langle y_{n+1}-m_{2}\left(y_{n}+1\right), m_{2}\left(y_{n+1}\right)+y_{n}-m_{2}\left(y_{n}\right)-y_{n+1}\right\rangle_{2} \\
& \geq\left\langle y_{n}-m_{2}\left(y_{n+1}\right), m_{2}\left(y_{n+1}\right)+y_{n}-m_{2}\left(y_{n}\right)-y_{n+1}\right\rangle_{2} \\
& -\rho_{2}\left\langle N_{2}\left(A_{2} y_{n}, B_{2} y_{n}\right)+M_{2}\left(C_{2} x_{n}, D_{2} y_{n}\right), \eta_{2}\left(m_{2}\left(y_{n+1}\right)+y_{n}-m_{2}\left(y_{n}\right), y_{n+1}\right)\right\rangle_{2} \\
& +\rho_{2}\left[b_{2}\left(y_{n}, y_{n+1}\right)-b_{2}\left(y_{n}, m_{2}\left(y_{n+1}\right)+y_{n}-m_{2}\left(y_{n}\right)\right)\right] .
\end{aligned}
$$

Then repeating the method, we have

$$
\begin{gathered}
\left\|y_{n}-y_{n+1}\right\|_{2} \leq \frac{1}{1-2 \tau_{2}}\left\{\left[1+\rho_{2} C_{2}+\delta_{2}\left(1+\sqrt{1-2 \rho_{2} \xi_{2}+\rho_{2}^{2}\left(\alpha_{2} \omega_{2}+\beta_{2} \gamma_{2}\right)^{2}}+\rho_{2} \mu_{2} \lambda_{2}\right)\right]\right. \\
\left.\left\|y_{n-1}-y_{n}\right\|_{1}+\rho_{2} \delta_{2} v_{2} \sigma_{2}\left\|x_{n-1}-x_{n}\right\|_{1}\right\} .
\end{gathered}
$$

From (16) and (21), we have

$$
\begin{aligned}
& \left\|x_{n}-x_{n+1}\right\|_{1}+\left\|y_{n}-y_{n+1}\right\|_{2} \\
& \leq\left\{\frac{1}{1-2 \tau_{1}}\left[1+\rho_{1} C_{1}+\delta_{1}\left(1+\sqrt{1-2 \rho_{1} \xi_{1}+\rho_{1}^{2}\left(\alpha_{1} \omega_{1}+\beta_{1} \gamma_{1}\right)^{2}}+\rho_{1} \mu_{1} \lambda_{1}\right)\right]+\frac{\rho_{2} \delta_{2} v_{2} \sigma_{2}}{1-2 \tau_{2}}\right\} \\
& +\left\|x_{n-1}-x_{n}\right\|_{1} \\
& +\left\{\frac{1}{1-2 \tau_{2}}\left[1+\rho_{2} C_{2}+\delta_{2}\left(1+\sqrt{1-2 \rho_{2} \xi_{2}+\rho_{2}^{2}\left(\alpha_{2} \omega_{2}+\beta_{2} \gamma_{2}\right)^{2}}+\rho_{2} \mu_{2} \lambda_{2}\right)\right]+\frac{\rho_{1} \delta_{1} v_{1} \sigma_{1}}{1-2 \tau_{1}}\right\} \\
& \quad \cdot\left\|y_{n-1}-y_{n}\right\|_{2} \\
& \leq \max \left\{\theta_{1}, \theta_{2}\right\}\left(\left\|x_{n-1}-x_{n}\right\|_{1}+\left\|y_{n-1}-y_{n}\right\|_{2}\right) .
\end{aligned}
$$

Where $\theta=\max \left\{\theta_{1}, \theta_{2}\right\}$,

$$
\theta_{1}=\frac{1}{1-2 \tau_{1}}\left[1+\rho_{1} C_{1}+\delta_{1}\left(1+\sqrt{1-2 \rho_{1} \xi_{1}+\rho_{1}^{2}\left(\alpha_{1} \omega_{1}+\beta_{1} \gamma_{1}\right)^{2}}+\rho_{1} \mu_{1} \lambda_{1}\right)\right]+\frac{\rho_{2} \delta_{2} v_{2} \sigma_{2}}{1-2 \tau_{2}},
$$$$
\theta_{2}=\frac{1}{1-2 \tau_{2}}\left[1+\rho_{2} C_{2}+\delta_{2}\left(1+\sqrt{1-2 \rho_{2} \xi_{2}+\rho_{2}^{2}\left(\alpha_{2} \omega_{2}+\beta_{2} \gamma_{2}\right)^{2}}+\rho_{2} \mu_{2} \lambda_{2}\right)\right]+\frac{\rho_{1} \delta_{1} v_{1} \sigma_{1}}{1-2 \tau_{1}} .
$$

By Condition (7), we have $\theta<1$. Therefore $\left\{\left(u_{n}, v_{n}\right)\right\}$ is a Cauchy sequence in $H_{1} \times H_{2}$. Let $\left(u_{n}, v_{n}\right) \rightarrow(u, v) \in H_{1} \times H_{2}$ as $n \rightarrow \infty$. Next, we claim that $(u, v) \in H_{1} \times H_{2}$ is a solution of SGNQVLI (1). In fact, by Theorem 2.1, we may assume that $(p, q) \in K_{1}(u) \times K_{2}(v)$ is the unique solution of SAGNQVLI (4), that is,

$$
\begin{aligned}
\langle p, u-p\rangle_{1} \geq & \langle x, u-p\rangle_{1}-\rho_{1}\left\langle N_{1}\left(A_{1} x, B_{1} x\right)+M_{1}\left(C_{1} x, D_{1} y\right), \eta_{1}(u, p)\right\rangle_{1} \\
& +\rho_{1}\left[b_{1}(x, p)-b_{2}(x, u)\right], \forall u \in K_{1}(x) .
\end{aligned}
$$

and

$$
\begin{aligned}
\langle q, v-q\rangle_{2} \geq & \langle y, v-q\rangle_{2}-\rho_{2}\left\langle N_{2}\left(A_{2} y, B_{2} y\right)+M_{2}\left(C_{2} x, D_{2} y\right), \eta_{2}(v, q)\right\rangle_{2} \\
& +\rho_{2}\left[b_{2}(y, q)-b_{2}(y, v)\right], \forall v \in K_{2}(y) .
\end{aligned}
$$

Similar argument as in proving (22), we have $\left\|x_{n}-p\right\|_{1}+\left\|y_{n}-q\right\|_{2} \leq \theta^{n}\left(\left\|x_{0}-p\right\|_{1}+\left\|y_{0}-q\right\|_{2}\right)$, where $\theta=\max \left\{\theta_{1}, \theta_{2}\right\}$, $\theta_{1}$ and $\theta_{2}$ is as above. It follows from Condition (7) that $\theta<1$, so $\left\|u_{n}-p\right\|_{1}+\left\|v_{n}-q\right\|_{2} \rightarrow 0(n \rightarrow \infty)$, this is, $p=u, q=v$.

Taking them into (23) and (24), we have

$\left\langle N_{1}\left(A_{1} x, B_{1} x\right)+M_{1}\left(C_{1} x, D_{1} y\right), \eta_{1}(u, x)\right\rangle_{1}-b_{1}(x, x)+b_{1}(x, u) \geq 0, \forall u \in K_{1}(x)$, $\left\langle N_{2}\left(A_{2} y, B_{2} y\right)+M_{2}\left(C_{2} x, D_{2} y\right), \eta_{2}(v, y)\right\rangle_{2}-b_{2}(y, y)+b_{2}(y, v) \geq 0, \forall v \in K_{2}(y)$.

That is, $(u, v) \in K_{1}(u) \times K_{2}(v)$ is a solution of SGNQVLI (1). This completes the proof.

\section{ACKNOWLEDGEMENTS}

This work was supported by the National Natural Science Foundation of China(11371070).

\section{REFERENCES}

[1] M A Noor, "Generalized set-valued mixed nonlinear quasi variational inequalities,” Korean J. Comput. Appl. Math. Vol.1,pp. 73-89,1998

[2] X P Ding, "Existence and algorithm of solutions for generalized mixed implicit quasi- variational inequalities,”Appl. Math. Comput. Vol.113, pp.67-80,2000

[3] Y L Zhao,Z Q Xia,L P Pang,L W Zhang, "Exostence of solutions and algorithm for a system of variational inequalities," Fixed Point Theory Appl. Vol.11,2010

[4] R Glowinski, J L Lions, R remolieres, "Numberical Analysis of Variational Inequalities,”North-Holland, Amsterdam ,1981

[5] M A Noor,K I Noor, "Some implicit methods for solving harmonic variational inequalities,” International J.Anal.Appl.Vol12(1),pp.10-14, 2016

[6] M A Noor,K I Noor, "Auxiliary principle technique for variational inequalities,” Appl. Math. Inf. Sci. Vol.11(1), pp.165-169,2017

[7] Z Liu,P P Zhang,J S Ume,S M Kang, “Auxiliary principle and iterative algorithm for generalized mixed nonlinear variational-like inequalities,” J. Nonlinear Sci. Appl. Vol.9,pp. 2959-2970,2016

[8] Y Q Qiu, J Z Chang,L C Ceng, “Auxiliary principle and iterative algorithm for a system of generalized set-valued strongly nonlinear mixed implicit quasi- variational-like inequalities,” J.Inequalities.Appl. Vol.38,pp.1-14,2016 